\title{
Eye movement research on the propositional structure of short texts
}

\author{
THEODORE S. MANDEL \\ University of Colorado, Boulder, Colorado 80309
}

\begin{abstract}
The present paper describes the application of computer-based programs to the evaluation of eye movements during reading of short texts. These programs operate in background, that is, after an experimental session is completed and the raw data are collected on magnetic tape. The first program reduces the raw data into a sequence of fixation locations and durations, eye movements, and eye blinks. A second program utilizes calibration coordinates and lineof-text information to transform the reduced data into a standard grid format. Fixation order, locations, and durations for each line of text read are output with respect to the line of text. The final program summarizes fixation locations, frequencies, and durations with respect to analyses of the texts into levels within a hierarchy of propositions in the text base (Kintsch, 1974).
\end{abstract}

Eye movement research in reading has been conducted since 1900 . The major problem with early motion picture eye movement measurement techniques was that data analysis involved tedious frame-by-frame analysis performed by hand. Recent eye movement measurement systems have incorporated microcomputers that track the position and pupil diameter of the reader's eye. The large amounts of data and the high rate of data collection of these new systems necessitate computerbased data collection and analysis that involves a fairly high level of programming sophistication.

This paper describes an eye movement monitor system used in the psychology department at the University of Colordao. Off-line computer programs are presented that perform two functions. First, raw eye movement data that are already collected and stored on magnetic tape are transformed into reduced data files consisting of fixation, eye movement, and eye blink information. Second, the reduced data are coordinated with the experimental text stimuli to produce output of fixation order, locations, and durations with respect to the line of text being read. These computer programs are written in FORTRAN. An additional program summarizes fixation information for a subject on each paragraph with respect to theoretical analyses of the texts into levels of propositions within a hierarchical text base.

\section{EXPERIMENTAL SYSTEM ORGANIZATION}

Figure 1 shows a schematic representation of the

The author wishes to acknowledge the assistance of all those associated with the eye movement monitor system, specifically, Dick Olson, Walter Kintsch, Peter Polson, and the CLIPR staff. Requests for reprints or for further information should be directed to Theodore S. Mandel, Department of Psychology, Muenzinger E-318, University of Colorado, Boulder, Colorado 80309. eye movement monitor system. The eye movement laboratory consists of a small room containing the complete Whittaker Model 1992S Eye View Monitor and TV Pupillometer System, remote-controlled slide projectors with Gerbrands shutters, a rear-projection screen, and additional videotape recorders and TV monitors. The illumination in the room can be varied from complete darkness to normal daylight illumination. An experimental subject is seated in an adjustable dentist chair and the head is held steady by a headstand with forehead and temple rests. The subject views a scene or text presented on a rear-projection screen while TV cameras photograph both the reader's left eye and the scene being viewed. The subject's eye rotation, and consequently, his point of fixation, is determined by the measurement of the center of the

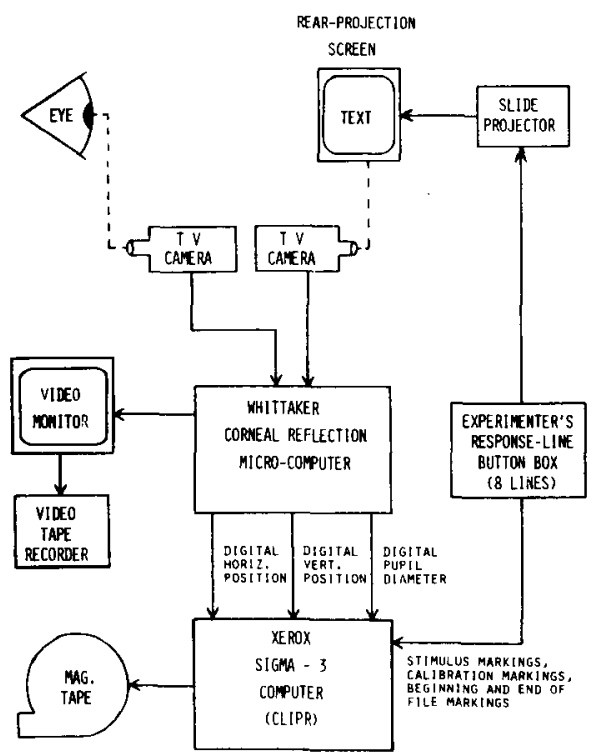

Figure 1. Schematic representation of eye movement monitor system. 
pupil with respect to the center of the corneal reflection. The center of the pupil and the center of the corneal reflection move together with head motion, but move differentially with eye rotation, hence the difference between their positions is indicative of the eye's point of fixation. In this way, eye position is independent of head position as long as the pupil image is contained within the field of view of the TV camera. A video monitor shows the experimenter an image of the scene being viewed by the subject. The continuous eye position information is presented as a spot or cross-hairs superimposed on the video monitor scene image. This video output can be videotaped for hand analysis or for later comparison with computer analysis of the data.

The microcomputer also outputs digital information that is passed on to the Xerox Sigma-3 computer in the Computer Laboratory for Instruction in Psychological Research (CLIPR), which is housed in the psychology department at the University of Colordao. Eye position is calculated as horizontal and vertical coordinates that are represented as 8-bit offset binary numbers. Pupil diameter is represented by a third 8-bit digital signal. These three signals are output with a positive clock pulse at a rate of 60 data points/sec. The sampling rate can be reset to average each two fields, providing output every $1 / 30 \mathrm{sec}$. Averaging the output provides quite adequate measurement for most experimental tasks and reduces the raw data storage by $50 \%$.

The entire experimental session is controlled by the experimenter from an eight-line response button box. The button box controls data collection by the computer and the slide projectors in use. One line starts data collection by the computer; a second button stops collection and generates an end-of-file mark on the magnetic tape. These two lines can be set so that data collection begins as a slide is projected and ends as a slide is removed or changed. Two slide projectors with automatic shutters are used so that the level of illumination on the viewing screen remains constant during slide changes and calibration and between trials. The remaining six response buttons can be used to mark the data by the experimenter or the subject. A foreground program on the CLIPR computer captures the incoming data, tags it with the time of day, and ships it out to magnetic tape, where it is stored as a series of six-integer records. The first two integers contain the time of day in milliseconds. The third, fourth, and fifth integers represent the horizontal eye position, vertical eye position, and pupil diameter, respectively. The sixth integer holds the response line buttonpresses. Thus, many experimental sessions can be stored sequentially on magnetic tape, as they can be easily marked with subject numbers, conditions, presentation orders, and special markings for experimental identification. The raw data stored on tape are then used as input for the following programs that are run in background to reduce the data and analyze eye fixation information for research on reading and text structure.

\section{REDUCTION PROGRAM DESCRIPTION}

A printout of the eye movement raw data records is presented in Figure 2. MS OF DAY is the value of the computer's running clock (in milliseconds) at the time of data capture, and DELTA T is the elapsed time between data points. These data were collected at the averaged sampling rate of 30 data points $/ \mathrm{sec}$. The next two columns represent the horizontal and vertical coordinates of the position of the eye. The coordinates are measured in TV scan lines and are values between -128 and +127 on both the horizontal and vertical axes. Therefore, when a subject is reading along a single line of text, the data show horizontal positions moving from negative positive values as the reader moves from left to right along the line of text. The values of the vertical coordinates should remain fairly constant as the reader scans along a single line of text. This normal sequence of eye positions in reading can clearly be seen in Figure 2, beginning with Line 13. Pupil diameter is also measured in TV scan lines. The values of the pupil diameter measure represent the number of scan lines that intersect the image of the pupil on the experimenter's TV monitor screen. Stimulus markings (not present in Figure 2) are numbers 1 to 8 that are added to the data record when a button is pressed on the response line button box.

The 43 raw data points shown here represent only $1.2 \mathrm{sec}$ of reading. Even with sentences or short paragraphs as experimental stimuli, it should be clear that printing the raw data and interpreting eye positions from these data are not worthwhile endeavors. For these reasons, it is necessary to reduce eye measurement data to a more use ful format.

Figure 3 presents a flowchart for the data reduction program. All algorithms for reducing eye measurement data are based upon the fact that eye movements are not continuous, but consist of pauses (fixations) around single locations separated by short periods of ballistic motion (eye movements). The algorithm for reducing eye movement data used in this program is based on a program developed by Mason (Note 1). It is assumed that only the duration and location of a fixation are desired. Saccades between fixations are classified as either eye movements or blinks, and microsaccadic movements around a fixation point are treated as noise and are averaged in with the fixation.

There are four parameters in this program, and their values depend upon the experiment and the degree of accuracy required by the experimenter. The first two parameters, DELTA HORIZ and DELTA VERT, determine the size of the window around a fixation 


\begin{tabular}{|c|c|c|c|c|}
\hline MS OF JAY & JELTA $T$ & $H J \backsim I Z O A \cdot T \Delta L$ & VEFTICAL & PUPIL \\
\hline 114212014. & 33 & -28 & 56 & 105 \\
\hline 114212547 & 33 & -67 & 97 & 91 \\
\hline 114212581. & 34 & -82 & 42 & 107 \\
\hline $1: 4212614$. & 33 & -94 & 42 & 107 \\
\hline 114212647. & 33 & -97 & 38 & 107 \\
\hline 114212681 . & 34 & -94 & -26 & 82 \\
\hline $11+21 \hat{c} / 1+$ & $\equiv 3$ & -114 & -59 & $c$ \\
\hline 114212747 & 33 & -128 & -59 & 0 \\
\hline 114212753. & 33 & -128 & .59 & 0 \\
\hline $1: 42128140$ & 34 & 7 & -99 & 61 \\
\hline 124212847 & 33 & -5 & -21 & 95 \\
\hline 114212880 & 33 & -69 & 35 & 109 \\
\hline $114212914^{\circ}$ & 34 & -104 & 45 & 108 \\
\hline 114212947 , & 33 & -110 & 42 & 108 \\
\hline 114212980. & 33 & -109 & 38 & 107 \\
\hline 124213013. & 33 & -100 & 38 & 108 \\
\hline 114213047 & 34 & -109 & 38 & 109 \\
\hline 114213080. & 33 & -110 & 38 & 109 \\
\hline 1142131130 & 33 & -107 & 42 & 109 \\
\hline 114213147. & 34 & -105 & 42 & 110 \\
\hline 114213180. & 33 & $-10 \bar{z}$ & 42 & 111 \\
\hline 114213213. & 33 & -103 & 42 & 111 \\
\hline $114 \leq 13246$. & 33 & -102 & 45 & 111 \\
\hline 114213280. & 34 & -103 & 42 & 112 \\
\hline 114213313. & 33 & -105 & $4 \hat{c}$ & $11 \bar{c}$ \\
\hline 114213346. & $3 \mathbf{3}$ & -107 & 45 & 112 \\
\hline 114213380. & 34 & -108 & 45 & 112 \\
\hline 114213413. & 33 & -103 & 45 & 112 \\
\hline 114213446. & 33 & $-10 C$ & 42 & 113 \\
\hline 114213480. & 34 & -98 & 42 & 113 \\
\hline 114213513. & 33 & -97 & 42 & 112 \\
\hline 1142135460 & 33 & -94 & 42 & 113 \\
\hline 114213573. & 33 & -94 & $4 \hat{\tilde{z}}$ & 112 \\
\hline 114213613. & 34 & -86 & 38 & 112 \\
\hline 114213040. & 33 & .71 & 35 & 113 \\
\hline 184213679. & 33 & -74 & 38 & 113 \\
\hline 1142137130 & 34 & -73 & 35 & 113 \\
\hline 114213746 & 33 & -71 & 38 & 113 \\
\hline 114213779. & 33 & -69 & 38 & 113 \\
\hline 114213812. & 33 & -68 & 35 & 112 \\
\hline 114213846 & 34 & -66 & 38 & 112 \\
\hline 114213879. & 33 & -68 & 38 & 112 \\
\hline 114213912. & 33 & -44 & 28 & 111 \\
\hline
\end{tabular}

Figure 2. Computer printout of raw data records.

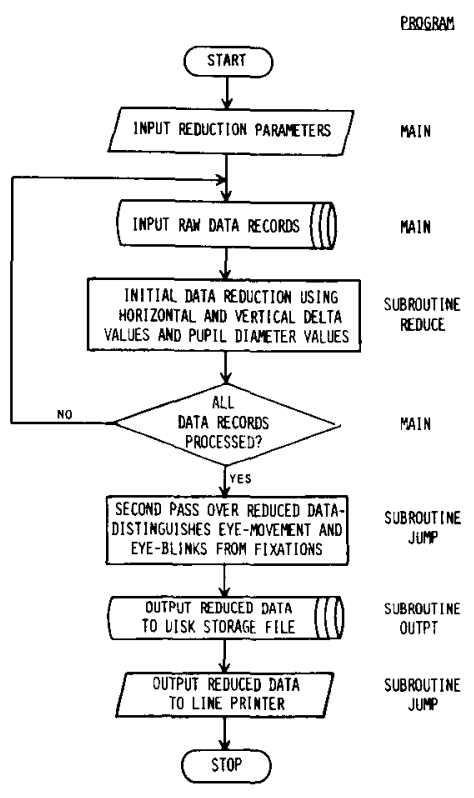

Figure 3. Flowchart for data reduction program.

point. Here, they are set at 10 scan lines, which represents a foveal angle of approximately $1 \mathrm{deg}$. DELTA MOVE is experimenter determined and represents the minimum duration value for actual fixations to occur. Any fixation whose duration is less than this value is tagged as an eye movement. In this research, DELTA MOVE is set for a minimum fixation duration of $133 \mathrm{msec}$. The reading literature shows fixations during normal reading range from approximately 150 to $300 \mathrm{msec}$. A pupil diameter delta value (DELTA PUPIL DIAMETER) is utilized to distinguish between eye movements and eye blinks. When an eye blink occurs, the pupil diameter measure falls greatly and often reaches zero (this is shown in the first 12 records of Figure 2). When a pupil diameter value falls more than the delta value below the average pupil diameter, an eye blink rather than an eye movement is recorded. The pupil diameter delta value is set at $40 \mathrm{TV}$ scan lines. These four reduction parameters are input in the main program. Each raw data record is read in singly and sequentially by the main program, thus duplicating real-time data collection conditions. 
The data reduction is performed by subroutine REDUCE using the horizontal and vertical delta values and the pupil diameter delta value. This subroutine basically compares the horizontal and vertical location values of a new data point with the values of a previous fixation or data point. When both the horizontal and vertical coordinates fall within the delta ranges, the new location values are averaged into the previous fixation. If the new location values differ from the previous ones by more than either of the horizontal or vertical deltas, the new point is designated as the beginning of a new fixation. Whenever a new data point is designated as a new fixation, the two previous fixations are checked to determine if their averaged horizontal or vertical coordinates differ by more than the delta values. If the differences are larger than the delta values, the subroutine continues. When both horizontal and vertical differences are less than the delta values, the two fixations are averaged together into a single fixation. This procedure is followed to correct for small microsaccades within a fixation that by chance exceed the delta values, thus triggering a new fixation. Without this checking procedure, two fixations would be recorded within the delta ranges for a single fixation point whenever any one of the data points within a single fixation exceeds either of the delta values. The pupil diameter delta parameter is used in this subroutine to differentiate eye blinks from fixation data. If any of the data points within a fixation fall below the delta value from the average pupil diameter, the fixation is tagged as an eye blink. The data reduction process continues with the main program presenting the next data point to subroutine REDUCE.

Once all data points in a specified data file have been processed by the reduction subroutine, then subroutine JUMP makes a second pass over the initially reduced data. This subroutine compares the fixation durations with DELTA MOVE. Any fixations whose durations are less than DELTA MOVE are denoted as eye movements. Those fixations previously tagged as eye blinks are also labeled and distinguished from actual fixations. After the second pass over the reduced data is complete, the reduced data are stored on magnetic disk (by subroutine OUTPT) and/or output to the line printer of the computer. The computer printout of the reduced data is shown in Figure 4.

The values of the reduction parameters are output with the program headings. The duration (DELTA T), horizontal and vertical eye positions, and pupil diameter are output for each fixation. Eye movements and eye blinks between fixations are output with their durations. All the raw data presented in Figure 2 are shown in reduced form in Figure 4. The raw data are now recorded as the sequence of an eye blink, a fixation whose duration is $632 \mathrm{msec}$, an eye movement, followed by another fixation whose duration is $266 \mathrm{msec}$, and a short eye movement. In Figure 4 there are fixations that are recorded as not having a saccadic eye movement between them. This result may occur when the fixations are close together and the saccadic duration is around $33 \mathrm{msec}$. The data collection may not capture a separate data point for eye movements of this duration. Eye movement and eye blink data between fixations are not analyzed in this research, but this information is presented in the reduced data output to give a clearer view of the reader's behavior at this level in the reading process. This program provides a considerable reduction of the raw data, typically a reduction of about 10 to 1 . If the data are reduced to fixation information without regard to eye movements or eye blinks, the reduction improves to a factor of 20 to 1.

\section{PRINT TEXT WITH FIXATION PROGRAM DESCRIPTION}

The reduced data output is first inspected by hand, and boundaries between fixations on separate lines of the text are established. This is accomplished by following the left-to-right reading scanning pattern within a line in the reduced data, and also by checking the reduced data with the videotaped data. This procedure is followed because the print text with fixations program was initially designed to present

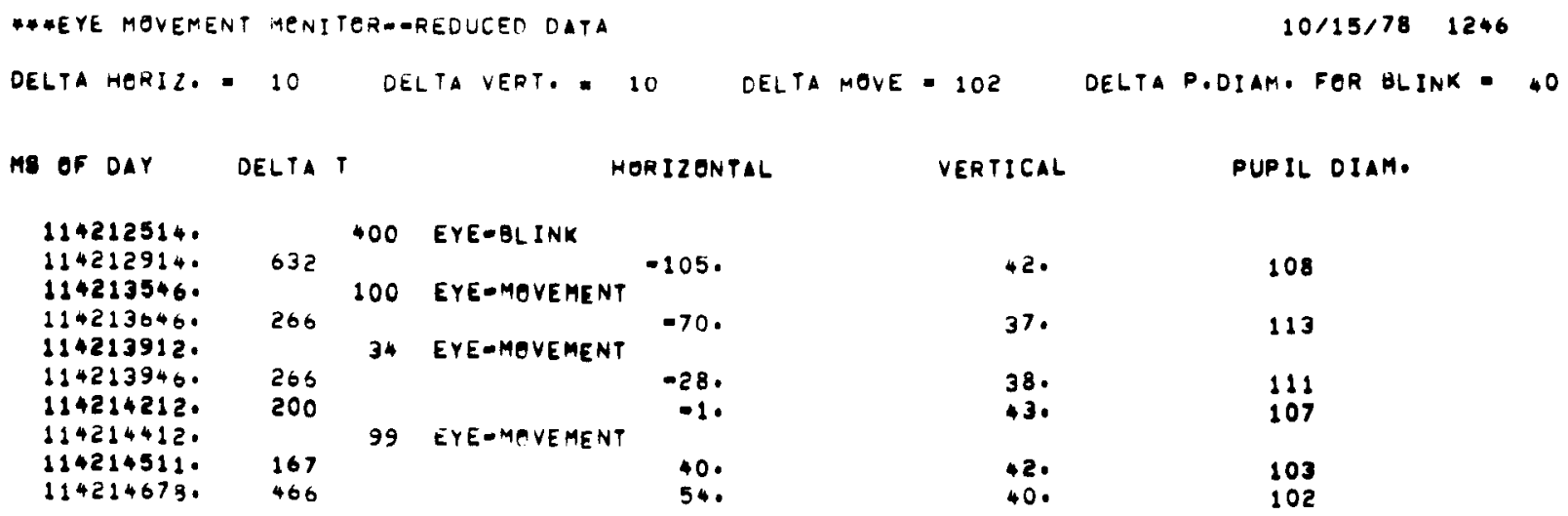

Figure 4. Computer printout of data reduction program. 
paragraph. During an experimental session, a subject is presented the five-point grid on a blank screen and is told to look at the center (1), the top left corner (2), the top right corner (3), the center again (1), the bottom left corner (4), the bottom right corner (5), and back to the center (1). The numbers in parentheses are the stimulus markings given to each fixation on that calibration point. The calibration values are then averaged to produce three calibration values along the horizontal axis. The left point is computed by averaging the horizontal values of Positions 2 and 4 (top left and bottom left corners). The center point is computed by averaging all three fixations at Position 1 , and the right point is computed by averaging the horizontal values at Positions 3 and 4 (top right and bottom right corners). The averaged calibration points are standardized by shifting them so that the center point is at 0,0 . This calibration procedure is shown in Figure 6 . Here, the original calibration positions and fixation locations are printed out, followed by the averaged and standard calibration points. The XCALIB and YCALIB values are simply the deviations of the averaged calibration center position from the zero center position.

Gridding factors are derived for data points left of center (XGRID LEFT) and points right of center (XGRID RIGHT) by dividing constant grid values $(-104$ and +104 , respectively) by the standard left and right calibration points. Three parameters that are input in this subroutine are TEXT STIMULI SHIFT, LEFT POINT CORRECTED, and RIGHT POINT CORRECTED. The TEXT STIMULI SHIFT parameter corrects horizontal position discrepancies between the viewing screen presentation of the calibration slide with the text stimuli slide. The LEFT POINT CORRECTED and RIGHT POINT CORRECTED parameters are input by the experimenter if the calibration data are not accurate with respect to the text. These values, if they are not zero, replace the standard grid calibration left and right points, respectively.

Linear regression equations, commonly used in calibration routines, are not necessary in this calibration subroutine because the data have already been divided into blocks of fixations per line. The grid factors are applied only to the horizontal coordinates of the reduced data fixations to map them onto a single line of text. The vertical coordinates are shifted according to YCALIB, but it is not necessary to apply grid factors to them to analyze fixation positions along each line of text. Two grid factors are derived because horizontal gain and linearity may vary for eye movements to the left of center and to the right of center.

Subroutine TEXT outputs in standard format the line of text corresponding to the block of fixations being processed (Figure 7). The reduced data records for the line of text are then input (subroutine FIXATE). In this subroutine, the horizontal coordinate of each fixation is first centered with respect to the standard grid by adding XCALIB. This value, if less than zero, is multiplied by XGRID LEFT to produce a gridded coordinate. If the horizontal value is greater than zero, it is multiplied by XGRID RIGHT. The order, position, and duration of each fixation within the line is then printed out under the line of text. Subroutine OUTPT produces a summary table of the fixation information for that line of text. The vertical coordinate of each fixation is shifted to the standard grid by adding

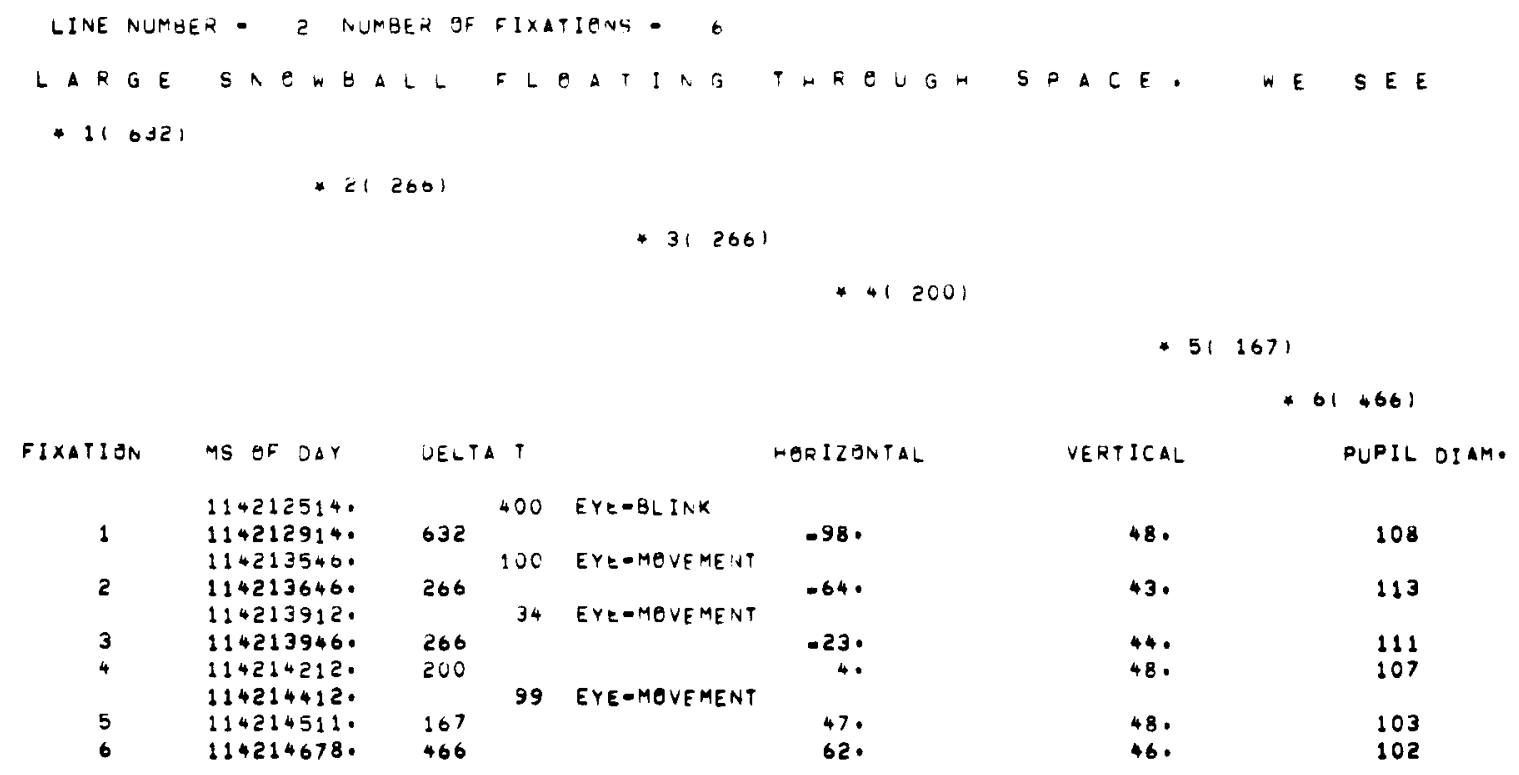

Figure 7. Computer printout of text with fixations program. 
YCALIB. The complete output for one line of text is presented in Figure 7. The position and order of a fixation are denoted by an asterisk (*) at the exact location followed by the order of fixation within the line. The duration of each fixation is given in parentheses. Each character of the text on the printout approximately equals two TV scan lines.

The eye movement raw data in Figure 2 have been transformed into a sequence of fixations, eye movements, and eye blinks. These data have been accurately mapped onto the text material that was viewed by a subject during an experimental reading session.

\section{THEORETICAL ANALYSIS}

The eye movement data resulting from the computer programs described here can now be further analyzed with respect to any type of reading measure. The total reading time can be broken down into total fixation time and eye movement or eye blink time. Fixation locations can be analyzed as to whether they fall on content or function words. Fixation frequencies and durations can be analyzed as a function of the general level of difficulty of the texts. These measures have already been inspected many times in the eye movement literature, and further analyses of this type are not of interest here. This research represents an initial step toward relating the eye movement patterns of readers to psychologically relevant features of text materials. More sophisticated eye movement measurement techniques and recent theories in linguistics and psychology on text structure and the representation of meaning of texts have led to explorations in this area of eye movement research.

The goal of the eye movement research presented here is to study the eye movement behavior of readers within the context of the semantically relevant units found in Kintsch's (1974) theory, that is, propositional text structure. Specifically, we are seeking to find out if the frequency of fixations on particular areas of the text correlate with our theoretically derived analysis of the text into hierarchical levels of meaning and importance to the text. Our hypothesis is that the frequency and duration of fixations will be greater on thse areas of the text analyzed as higher levels with respect to meaning and importance. Areas of the text that are in lower levels in the text hierarchy should receive fewer fixations and also fixations of shorter durations. Regressive fixations between lines of text are also an important variable in the reading process. Often, a reader regresses to a word that ties other information together, that is, a word that is necessarily important for comprehension of the text. We therefore predict that regressive fixations will also follow the levels hypothesis. The computer programs presented here transform the unanalyzable raw data output of the eye monitor system into a form that is compatible with the text itself and our theoretical analysis of the text material. The experimental procedures involve silent reading of 1470 -word paragraphs on either history or science topics, with immediate verbal recall after each paragraph. The general experimental procedures follow closely the experiments in Kintsch, Kozminsky, Streby, McKoon, and Keenan (1975).

Figure 8 presents the tabular output of a computer program that summarizes the fixation information from a subject's reading of one paragraph. There are five levels in the propositional text structure of this particular paragraph. The numbers in the top right corner represent the total number of fixations on the paragraph and the total duration of these fixations. The number of propositions and words per level in the theoretical analysis of the text are given on the right of the summary table. Listed under the heading "Fixations" are the number of fixations and percent of total fixations per level. The durations are presented as total duration per level, percent of total paragraph duration, and average duration per fixation for each level. The number of fixations and total duration per level are then divided by the number of words per level

\begin{tabular}{|c|c|c|c|c|c|c|c|c|c|c|c|c|}
\hline \multirow[b]{2}{*}{ LEVEL } & \multirow{3}{*}{ P. 01} & ARED & LEVELS & \multicolumn{2}{|c|}{ ANALYIS } & & & \multirow[b]{2}{*}{ CURATION/WORD } & \multirow[b]{2}{*}{ FIX IPROP. } & \multirow[b]{2}{*}{ DURATION/PRO } & \multirow[b]{2}{*}{ P. FIX: } & \multirow[b]{2}{*}{ D. DURATION } \\
\hline & & $F\lfloor x$ & ATIONS & DUFATI & $\operatorname{lan}_{0-\infty}$ & SEC.1 & $f: x=/$ WORD & & & & & \\
\hline & & & $x$ & TSTAL & $x$ & MEAN & & & & & 168. & 49399. \\
\hline 1 & 110 & 19 & .11 & 6522 & .13 & 343 & 3.8000 & 1304 & 19.0000 & 6522 & w. 5 & $p=$ \\
\hline 2 & 201 & 49 & $\cdot 29$ & 14078 & .28 & 297 & 1.7500 & 502 & 6.1250 & 1759 & W. 28 & $p=$ \\
\hline 3 & 97 & 59 & $\cdot 35$ & 16945 & .34 & 287 & 1.4750 & 423 & 4.9167 & 1412 & $w=40$ & $p=12$ \\
\hline 4 & 98 & 36 & .21 & 10223 & .21 & 283 & 1.4400 & 408 & 4.0000 & 1135 & $\mathrm{H}=25$ & Pe \\
\hline $\mathbf{B}$ & 91 & 5 & .03 & 1631 & .03 & 326 & 0.8333 & 271 & 2.5000 & 18 & $w=b$ & $\rightarrow 2$ \\
\hline$\bullet$ & 0 & 0 & .00 & 0 & .00 & 0 & 0.0000 & 0 & 0.0000 & 0 & $w=0$ & $P 0$ \\
\hline 7 & 0 & 0 & .00 & 0 & .00 & 0 & 0.0000 & 0 & 0.0000 & 0 & we & $p=$ \\
\hline
\end{tabular}

Figure 8. Computer printout of levels analysis program. 


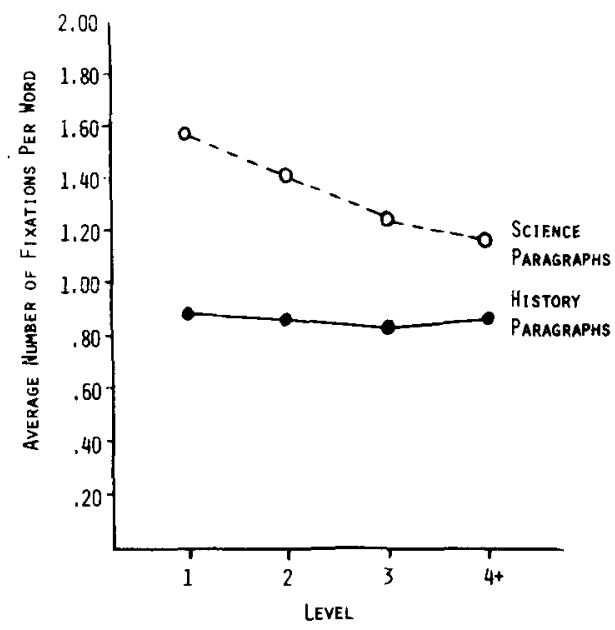

Figure 9. Average number of fixations per word as a function of level in the text base for history and science paragraphs.

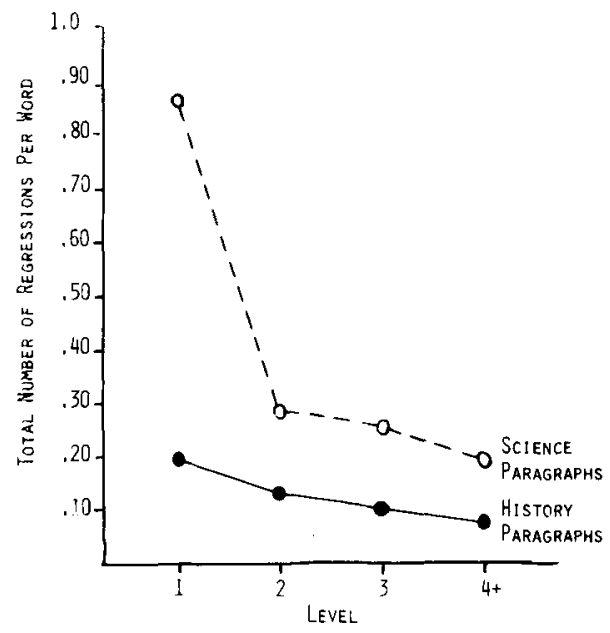

Figure 10. Total number of regressions per word as a function of level in the text base for history and science programs.

and the number of propositions per level to produce the rest of the summary table.

The fixation data for five subjects are shown in Figure 9. History paragraphs show no levels effect, while science paragraphs show a marginal levels effect. Figure 10 presents the regression data for all subjects. Here, both history and science paragraphs show a significant levels effect. Regressions to words at the superordinate level (Level 1) outnumber regressions at the subordinate levels (Level 4+) by a factor of 2 for history paragraphs. The science paragraphs show a strong $4: 1$ ratio between regressions at the superordinate level and regressions at the subordinate levels.

These results show that eye movement data can be utilized in the study of processing activities during reading. The relationship between eye movement behavior of readers and the underlying semantic features of texts is now being investigated by numerous researchers (Carpenter \& Just, 1978; Rothkopf \& Billington, Note 2; Shebilske, Note 3). The development and improvement of computer programs to reduce and analyze the high-speed collection of eye movement data such as those described in this paper will greatly facilitate theoretical research on eye movement behavior during reading.

\section{REFERENCE NOTES}

1. Mason, R. L. Digital computer estimation of eye fixations. Paper presented at the 5th Annual Meeting of the National Conference on the use of On-Line Computers in Psychology, November 5, 1975.

2. Rothkopf, E. Z., \& Billington, M. J. Goal-guiding learning from text: Inferring a descriptive processing model from inspection times and eye movements. Manuscript submitted for publication, 1979.

3. Shebilske, W. L. Reading eye movements, macrostructure, and comprehension processes. Paper presented at a conference on "Processing of Visible Language." Eindhoven, The Netherlands: Institute for Perception Research (IPO), September 5-8, 1977.

\section{REFERENCES}

Carpenter, P. A., \& Just, M. A. Reading comprehension as the eyes see it. In M. Just \& P. Carpenter (Eds.), Cognitive processes in comprehension. Hillsdale, N.J: Erlbaum, 1978.

KINTSCH, W. The representation of meaning in memory. Hillsdale, N.J: Erlbaum, 1974.

Kintsch, W., Kozminsky, E., Strehy, W. J., McKoon, G., \& KeEnAN, J. M. Comprehension and recall of text as a function of content variables. Journal of Verbal Learning and Verbal Behavior, 1975, 14, 196-214. 